\title{
Pattern changes in rapid serial visual presentation tasks without strategic shifts
}

\author{
JUAN BOTELLA \\ Universidad Autonoma de Madrid, Madrid, Spain \\ and \\ CHARLES W. ERIKSEN \\ University of Illinois, Urbana-Champaign, Illinois
}

\begin{abstract}
Observed patterns of intrusions in rapid serial visual presentation (RSVP) tasks have been used to infer whether relevant stimulus features are processed in serial or parallel. It has been claimed that intrusion patterns that consist primarily of posttarget stimuli result from the serial processing of stimulus features, whereas symmetrical patterns of intrusions result from the parallel processing of stimulus features. However, Botella and Eriksen (1991) have claimed that the parallel processing of stimulus features can generate both types of intrusion patterns. Here we report on an RSVP task in which the interstimulus interval (ISI) was manipulated randomly in order to prevent subjects from adopting a different strategy for each ISI. The subjects produced both patterns of intrusion.
\end{abstract}

In a typical rapid serial visual presentation (RSVP) task, a series of stimuli is presented at a very high rate in a single spatial location. The subjects are instructed to report some characteristic (response dimension) of the only item that contains a specified characteristic (key dimension). For example, Lawrence (1971) asked subjects to report the identity (response dimension) of the only word presented in uppercase (key dimension); McLean, Broadbent, and Broadbent (1983, Experiment 1) asked subjects to report the color of a specified letter in one condition, and in the other to report the identity of the letter that had a specified color (reversed roles of color and shape as key and response dimensions).

The most interesting result of such experiments is that often the subjects report some feature belonging to the response dimension that, in fact, has been presented in the series, but not in the target; these intrusions from filler items, or incorrect combinations of features really presented, are sometimes called illusory conjunctions (Treisman \& Gelade, 1980). Moreover, the features incorrectly reported come from items presented after the target (posttarget intrusions) more frequently than from items presented before the target (pretarget intrusions). However, sometimes a balance between both types of intrusion is found, and, in addition, Intraub (1985) found more prethan posttarget intrusions.

The research reported here was supported for Juan Botella by a grant from the Ministerio de Educacion y Ciencia of Spain and for Charles W. Eriksen by Public Health Service Research Career Program Award K6-MH-22014 and Public Health Service Research Grant MH-012006. Requests for reprints should be sent to Juan Botella, Facultad de Psicología, Universidad Autonoma de Madrid, 28049 Madrid, Spain.
Two main interpretations of the typical posttarget pattern have been formulated. One is the theory proposed by Broadbent and his colleagues (Broadbent, 1977; McLean et al., 1983). From their point of view, the subjects perform two serial operations-the detection of the feature that defines the target, and the identification of the response dimension of that item. Sometimes the detection takes too much time, and the identification process is performed on the item presented just after the target. Since in some experimental conditions the pattern of intrusions found has been symmetrical, they argue that the subjects have two modes of processing available, one serial and the other parallel, and that the subjects select the mode most adapted to the task demands. A serial strategy would produce patterns of predominance of posttarget intrusions, and a parallel strategy would produce symmetrical patterns (Gathercole \& Broadbent, 1984; McLean et al., 1983).

An alternative explanation has been formulated by Botella and Eriksen (1991). It is based on the parallel processing of the key dimension and the response dimension, and on the serial selection of the outputs from those processes. The patterns of intrusions would depend on the average time of processing for both dimensions (the key and response dimensions), and on the rate of presentation of the stimuli. From this perspective, the three possible kinds of patterns (posttarget, symmetrical, and pretarget) can be generated by the same system, by changing the level of difficulty of the discriminations and/or the interval of presentation.

Botella and Eriksen (1991), who have reviewed the evidence favoring the serial and parallel processing positions, have concluded that there is stronger evidence to support a parallel view. However, whether different patterns of 
results can be obtained without strategic shifts of processing mode has not been addressed. The present experiment suggests that the system is able to generate different patterns without strategic shifts.

One of the arguments of Gathercole and Broadbent (1984) is apparently based on the assumption that the posttarget pattern intrusions are only compatible with a serial mode of processing, and that symmetrical intrusions are only compatible with a parallel processing mode; therefore, to infer the mode of processing adopted in each particular experimental condition, they use the kind of pattern observed. For them, it is the assessment made by the subject of the level of difficulty of the task that determines the adopted processing mode. Yet, on the contrary, a parallel model can produce different intrusion patterns; this has been shown on a more theoretical level by Botella and Villar (1989). They performed a Monte Carlo simulation of a simple version of a parallel model; the model generated different patterns of intrusions, due to manipulation of the average processing times of both key and response dimensions, as well as the rate of presentation. However, there is no empirical evidence that the same results occur when real subjects are prevented from consistent strategic shifts.

In the present experiment, an RSVP task with colored letters was used. The key dimension consisted of the color of the stimulus, and the response dimension consisted of stimulus shape. The main independent variable was the interstimulus interval (ISI). Trials with different ISIs were mixed randomly within a block in such a way that the subjects could not predict how difficult the next trial would be. Therefore, the processing mode had to be the same for all conditions. The parallel model predicts that significant changes in the patterns of intrusions can be produced in this situation.

Another independent variable, with an exploratory character, was also included. This was the separation between the ISI and the exposure time of the items. Usually in this kind of experiment, each item is replaced by the next in such a way that the exposure for each item equals the ISI (these we will call the classical conditions). The variable of separation was included to explore what happens when the items are presented for a shorter duration than the ISI.

\section{METHOD}

\section{Subjects}

Fifteen undergraduate students with normal or corrected-to-normal vision were paid for their participation. All these subjects had participated in Experiment 1 of Botella and Eriksen (1991), so they already had a good deal of practice with the task and the procedure.

\section{Stimuli and Materials}

The stimuli were presented on a Zenith CRT connected with and controlled by a Zenith Data System computer. Each list had 17 items; the 1 st and last 2 were black numbers, whose presence was known to the subjects. Their function was to obviate the well-known tendency of subjects in such tasks to frequently report the first and last items in the series. The letters contained in each list consisted of 13 different uppercase consonants, selected from the alphabet randomly. These letters were in five colors: white, yellow, green, red, and blue (the computer's standard colors). On each list, one target letter was designated that occurred with equal probability in the 8th, 9th, or 10th position on the list. The target letter appeared in the color that would be the key feature in that list, and this color was not used for any other item on the same list. The five colors were used as the target-defining feature equally often over the lists. The 2 items before and after the target (Positions -2 , $-1,+1$, and +2 ) were constructed with the other four colors, without repetitions. The remaining items were also colored with these four colors randomly, with the restriction that 2 consecutive items never share the same color. The background color was dark gray. The letters subtended $0.64^{\circ}$ in height and $0.29^{\circ}$ in width.

\section{Procedure}

The subjects were located $40 \mathrm{~cm}$ from the screen. At the beginning of each trial, the screen displayed the question, "Which letter is in this color?" alongside of this question appeared a color patch with the color that would be the key feature of the target on that trial. The patch appeared at the same location as that at which the item series would appear. When a subject pressed the keyboard space bar to begin the trial presentation, the question disappeared, and the colored patch was replaced by a black cross (fixation point) for $500 \mathrm{msec}$; the cross was followed by the first item in the series. After the last item in the series, a response menu was presented, with nine numbered alternatives. The first seven alternatives were the letters from the critical set (the target, the three letters presented just before, and the three letters presented just after), all in black, and in a random order. The eighth alternative was, "I don't know"; the ninth alternative was, "not in the menu."

The experimental conditions were defined by the stimulus exposure time and the empty interval between them. In the classical conditions, the exposure time equaled the ISI (each item was replaced by the next). In the other conditions, the ISI was longer than the exposure duration. We will use time in milliseconds to designate the exposure time and empty interval for each condition; the 12 conditions were as follows: $66 / 0,66 / 16,66 / 33,66 / 50,83 / 0,83 / 16,83 / 33,100 / 0,100 / 16,100 / 33$, $116 / 0$, and $116 / 16$. During the empty interval, the position where the series was being presented was the same color as the rest of the screen (dark gray).

The subjects received seven blocks of 65 trials each during a single session of about $1 \mathrm{~h}$. The first 5 trials of each block were eliminated from data analysis because they were considered a warm-up. The other 60 trials of each block contained 5 trials from each of the 12 conditions in a random order. The first block was eliminated, because it was considered practice. Therefore, 30 trials from each condition were collected. The responses were classified according to the position occupied in the series by the letter chosen as the response. Between each two blocks, the subjects rested 2 or $3 \mathrm{~min}$.

\section{RESULTS}

For each subject, the percentage of choices of each response alternative was calculated for each experimental condition. However, in order to do statistical analysis over the positional responses, the data were transformed. The reason for this transformation is that with the percentages calculated over the total number of trials, the different conditions were not completely comparable, because the numbers of "I don't know" and "not in the menu" responses were very different between the ISI conditions (from $18.8 \%$ for ISI $=66$ to $2.6 \%$ for ISI $=116$ ). Thus, before analysis, the percentages including only positional responses were recalculated. The results for the four classical conditions (that is, with exposure time $=$ ISI) will be described first, and then the effects of the empty interval will be analyzed.

The first four rows of Table 1 show the percentage of responses for the four classical conditions, and the ratios 
Table 1

Average Percentage of Choices of the Response Feature Belonging to Each Position in the Central Set

\begin{tabular}{|c|c|c|c|c|c|c|c|c|}
\hline \multirow[b]{2}{*}{ Condition } & \multicolumn{7}{|c|}{ Relative Position of the Feature Reported } & \multirow{2}{*}{$\begin{array}{l}\text { Ratio } \\
+1-\end{array}$} \\
\hline & -3 & -2 & -1 & 0 & +1 & +2 & +3 & \\
\hline \multicolumn{9}{|c|}{ Classical Conditions } \\
\hline $66 / 0$ & 4.9 & 3.0 & 10.9 & 42.3 & 26.6 & 7.9 & 3.5 & 2.02 \\
\hline $83 / 0$ & 0.6 & 6.4 & 7.3 & 57.8 & 21.8 & 3.5 & 2.6 & 1.95 \\
\hline $100 / 0$ & 1.1 & 3.6 & 12.7 & 68.1 & 11.5 & 1.1 & 1.8 & 0.83 \\
\hline $116 / 0$ & 0.4 & 0.8 & 3.6 & 90.0 & 3.4 & 0.4 & 1.3 & 1.06 \\
\hline \multicolumn{9}{|c|}{ Blank Interval Conditions } \\
\hline $66 / 16$ & 3.5 & 3.0 & 4.4 & 70.0 & 15.6 & 2.3 & 1.2 & 1.75 \\
\hline $66 / 33$ & 0.6 & 4.9 & 4.7 & 79.8 & 8.4 & 1.1 & 0.4 & 0.97 \\
\hline $66 / 50$ & 1.3 & 2.9 & 7.5 & 80.5 & 5.9 & 0.6 & 1.3 & 0.67 \\
\hline $83 / 16$ & 2.7 & 3.8 & 6.6 & 78.2 & 6.4 & 2.1 & 0.4 & 0.68 \\
\hline $83 / 33$ & 0.6 & 2.5 & 5.5 & 81.9 & 8.5 & 0.4 & 0.6 & 1.10 \\
\hline $100 / 16$ & 2.4 & 0.4 & 2.0 & 86.6 & 5.4 & 2.0 & 1.1 & 1.77 \\
\hline $100 / 33$ & 2.4 & 2.0 & 1.1 & 91.0 & 2.6 & 0.2 & 0.6 & 0.62 \\
\hline $116 / 16$ & 1.3 & 0.2 & 3.1 & 90.4 & 3.8 & 0.6 & 0.6 & 1.09 \\
\hline
\end{tabular}

Note-The last column contains the ratio between the posttarget and pretarget responses for each condition.

between post- and pretarget intrusions. The general level of performance, as measured by correct choices, was examined with an analysis of variance (ANOVA). It is clear in rows 1-4 in Table 1 that correct responses change with ISI $[F(3,42)=79.837, p<.0001]$.

A clear decreasing tendency in "I don't know" and "not in the menu" responses is observed as the ISI is increased. An ANOVA of the percentage of "I don't know" responses revealed a significant effect $[F(3,42)=10.613$, $p<.0001]$.

Intrusion patterns were analyzed with a sign test. ${ }^{1}$ Consistent with other research done using this paradigm, there was a significantly larger number of intrusions from adjacent positions than from the other positions when shorter ISIs were used. But for the longer ISIs, the difference between intrusions from adjacent and other positions was reduced. Within the distribution of adjacent intrusions, these were predominantly coming from the +1 position $(+1>-1)$ in conditions with shorter ISIs: ISI $=66$

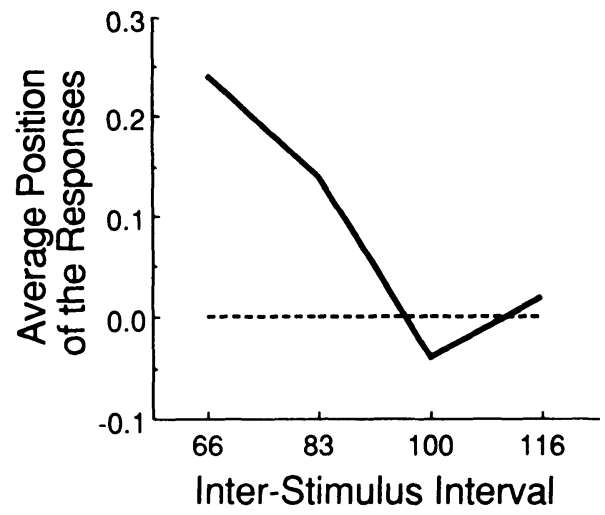

Figure 1. Average position of the response features reported by the subjects in the classical conditions.
$(13 / 13, p<.001)$, and ISI $=83(13 / 14, p<.001)$. Also, the general predominance of post- over pretarget intrusions (posttarget $>$ pretarget) was statistically significant in the same conditions: ISI $=66(13 / 15, p<.01)$, and ISI $=83(13 / 15, p<.01)$.

The posttarget/pretarget ratio value may change markedly with only a few deviant trials. When performance is at about $75 \%$ correct responses or below, the number of intrusions is less sensitive to sampling fluctuations. However, when the percentage of intrusions is less than $25 \%$, slight sampling fluctuations can produce misestimations of that ratio. A way to avoid this problem is to calculate the average position of the responses. This index is more stable because the correct responses have a heavier weight. The procedure is to assign a 0 to the correct responses, $-1,-2$, and -3 to pretarget intrusions coming, respectively, from items in those positions, and $+1,+2$, and +3 to posttarget intrusions, then calculating the average position of the reported features. After this index was calculated for each subject and each condition, an ANOVA was applied to the obtained values (Figure 1), and a main effect of ISI was found $[F(3,42)=5.716, p<.01]$.

With regard to the conditions with ISI longer than exposure time, the results show that the level of performance was clearly influenced by this manipulation, as can be observed in rows 5-12 in Table 1 . The only condition with an empty interval greater than zero that had a significant posttarget pattern was $66 / 16(12 / 14, p<.01)$. The other conditions showed more symmetrical patterns. Although in some conditions a $+/-$ ratio below 1 was found, there was no significant predominance of pre- over posttarget intrusions in any of the conditions.

In addition, there is an interesting result when some selected cross-comparisons are made. For example, conditions $66 / 16$ and $83 / 0$ shared the same ISI, but the first had a shorter exposure time than the second, and a short empty interval, while the second had no empty interval. The short empty interval improved the results (the same can be seen when 83/16 and 100/0 conditions are compared, but not when the $100 / 16$ and $116 / 0$ conditions are compared).

\section{DISCUSSION}

These results indicate, first, that without strategic changes by the subjects, significant changes can be found in the pattern of intrusions when only ISI is manipulated. Thus, because the same processing mode can generate different patterns, it is not appropriate to infer the mode of processing from the observed pattern; it is necessary to develop other methods (Botella \& Eriksen, 1991). Specifically, the results indicated that with the shorter ISIs there is a predominance of posttarget intrusions, but as both ISI and exposure time increase, the pattern becomes more symmetrical. For instance, when the exposure time was 66 or 83 , the pattern was clearly posttarget intrusion, but when the values were 100 or 116 , the pattern was clearly symmetrical. Furthermore, this change was not sharply demarcated; rather, it seems that it happened step by step with changes in ISI. This change in the intrusion pattern cannot be interpreted as a strategic shift in the processing mode, because the subjects did not have the information needed to make that decision before each trial.

Furthermore, Broadbent and his colleagues have suggested that the system shifts from a serial mode to a parallel mode when the difficulty of the task is increased (Gathercole \& Broadbent, 1984; McLean, Broad- 
bent, \& Broadbent, 1983). The present findings show, however, that the shift from a posttarget to a symmetrical pattern occurs when the task is made easier. Using Broadbent's logic, the conclusion would be that the subjects were using a parallel mode of processing features for the easiest conditions, and the serial mode for the more difficult conditions. This conclusion contradicts those reached in their experiments (Gathercole \& Broadbent, 1984).

Botella and Eriksen (1991) have proposed a model based on the temporal simultaneity of the feature's representation. The average times associated with the different key and response dimensions could produce pretarget, posttarget, or symmetrical patterns. However, Botella and Eriksen used evidence different from the pattern of intrusions to show that the information from the key and response dimensions is processed in parallel.

Second, it seems that the use of a brief blank interval between the stimuli does have an effect, although this effect is not yet clear. Originally, the question addressed was whether a reduction in the physical energy of the stimuli (via a decrement of the duration) would make it more confusable. The results do not show such an effect, but rather the opposite. When the ISI is 83 or $100 \mathrm{msec}$, it seems that performance is better if stimuli are presented at durations shorter than the ISI; a short empty interval $(16 \mathrm{msec})$ helps to improve performance. However, with a longer ISI (116 msec), the inclusion of a 16-msec empty interval does not improve performance; indeed the performance with this ISI is better if the stimulus is present throughout the ISI. With long ISIs, the empty interval does not reduce the occurrence of illusory conjunctions. It appears that something important happens when the duration of the stimuli exceeds the value of $100 \mathrm{msec}$

\section{REFERENCES}

Botella, J., \& Eriksen, C. W. (1991). Illusory conjunctions in rapid serial visual presentation tasks. Manuscript in preparation.
Botella, J., \& Villar, M. V. (1989). Modelos de Procesamiento en tareas de Presentacion Rapida de Series Visuales. Psicologica, 10, 179-203.

Broadbent, D. E. (1977). The hidden preattentive processes. American Psychologist, 32, 109-118.

Gathercole, S. E., \& Brondbent, D. E. (1984). Combining attributes in specified and categorized target search: Further evidence for strategic differences. Memory \& Cognition, 12, 329-337.

INTRAUB, H. (1985). Visual dissociation: An illusory conjunction of pictures and forms. Journal of Experimental Psychology: Human Perception \& Performance, 11, 431-442.

LAWRENCE, D. H. (1971). Two studies of visual search for word targets with controlled rates of presentation. Perception \& Psychophysics, 10, 85-89.

Mclean, J. P., Broadbent, D. E., \& Broadbent, M. H. P. (1983). Combining attributes in rapid sequential visual presentation tasks. Quarterly Journal of Experimental Psychology, 35A, 171-186.

Treisman, A., \& Gelade, G. (1980). A feature integration theory of attention. Cognitive Psychology, 12, 97-136.

\section{NOTE}

1. The number of comparisons made is not always equal to the total number of subjects (15), because there were some ties; these were excluded from the analysis.

(Manuscript received June 25, 1990.) 